\title{
A Refreshed View of the Rumen Microbiome of Beef Cattle in Light of Next-Generation DNA Sequencing
}

\author{
Lourenco JM*, Fluharty FL and Callaway TR \\ Department of Animal and Dairy Science, University of Georgia, Athens, GA, USA
}

Submission: November 15, 2018; Published: December 03, 2018

"Corresponding author: Jeferson M Lourenco, Department of Animal and Dairy Science, University of Georgia Address: 425 River Road. Athens, GA, USA

\begin{abstract}
The rumen of cattle is densely populated by a myriad of diverse microorganisms. The bacterial proportion of this consortium has been elucidated regarding its ability to break down and metabolize complex compounds (particularly polysaccharides) which would otherwise be undigestible to the mammalian host. True symbioses such as that found in the rumen between the host animal and the resident ruminal microbial population is rare in nature. The development of Next-Generation DNA Sequencing techniques now allows us to study the microbial composition in greater detail than ever before. The implementation of new technology to characterize the ruminal microbial population, combined with animal performance data, has opened a new field of study for animal scientists and ruminant nutritionists. Ultimately, we must correlate animal feed efficiency and microbial degradative specific activities with the overall ruminal microbial diversity. These correlations will pave a new way for nutritionists to feed cattle more efficiently, precisely selecting in favor of the microbial populations that are most associated with efficient animal performance.
\end{abstract}

Keywords: Cattle; DNA; Feed efficiency; Microbiome; Next-generation sequencing

\section{Introduction}

The rumen is a blind sac in the digestive tract that functions as an effective system for the conversion of plant cell wall biomass into microbial protein, short chain fatty acids, and gases $[1,2]$. The ruminal microbiome of cattle is characterized by its high population density ( $>1010 \mathrm{CFU} / \mathrm{ml}$ ), wide diversity, and complex web of interactions [2,3]. Although cellulose is the most common carbohydrate on earth, it cannot be degraded by mammalian enzymes, however, the microbial population inhabiting the rumen provides this capacity, allowing the ruminant animal to occupy ecological niches that other animals cannot. Catabolism of plant cell walls is rarely carried out by a single species of microorganism; for example, the initial colonization of plant fiber is often performed by fungi that degrade the easily available side chains of complex polysaccharides, followed by another subset of bacteria that degrade the main chains of cellulose and xylan [1]. Because of the importance of the symbiotic relationship between ruminant animals and the microbial population of the rumen, characterization of their microbial community is very important.

However, traditional culture-dependent methods have not been able to elucidate the whole ruminal ecosystem, as many species are obligate anaerobes and/or do not thrive in laboratory conditions. For instance, although Ruminococci have been studied in many culture-based experiments [4], recent studies using modern molecular techniques have found that this genus is present at relatively low abundances in the ruminal microbiome $[5,6]$. In addition, members of the Ruminococcaceae family that have been identified through modern techniques may be different from the ones identified through culture-based techniques, and consequently have different biological functions and enzymatic activities [5]. Thus, new approaches such as next-generation DNA sequencing have contributed to a better understanding of the complete (and more accurate) rumen microbiome and have opened new arenas for study.

\section{Rumen Microbiome Composition and its Effects on} Animal Performance

Overall, ruminal microbial communities are dominated by members of the phyla Firmicutes and Bacteroidetes. Collectively, these two phyla comprise approximately $85 \%$ of the total ruminal bacterial population [5-8]. After performing pyrosequencing of 16S rRNA genes, Rosewarne et al. [7] found a predominance of sequences from the phyla Bacteroidetes (47.2\%) and Firmicutes (42.3\%). Similarly, McCann \& Myer et al. [5,8] reported prevalence of Bacteroidetes ranging from 53 to 78\%, and Firmicutes from 15 and $33 \%$ in ruminal samples.

The relationship between feed efficiency of cattle and the rumen microbial populations using next-generation sequencing has been investigated under distinct animal production scenarios. McCann [5] studied the correlation between rumen microbiome and feed-efficiency in Brahman (Bos indicus) bulls. Overall, the authors classified $67.5 \%$ of their sequences as Bacteroidetes, and $22.9 \%$ as Firmicutes, but neither of these two dominant phyla correlated with feed efficiency. However, at the family level, Prevotellaceae was the most abundant bacteria, and a 
greater presence of this family was detected in less-efficient bulls, compared to the most-efficient ones. Conversely, an undefined family of Bacteroides was greater in the most-efficient bulls. In another study, Myer et al. [8] fed a high-concentrate diet to a total of 345 steers for a period of 63 days and individually measured ADG and feed intake. Steers with distinct animal performance phenotypes were selected, and ruminal samples were sequenced using bacterial 16S rRNA gene amplicons. Overall, they found that the most efficient group of steers (i.e. high ADG with low feed intake) had a lower percentage of Bacteroidetes (mainly due to Prevotella) in their microbiome, compared to less-efficient steers. In contrast, Firmicutes was positively correlated with higher feed efficiency.

Furthermore, within this phylum, the genera Dialister had a significantly greater percentage in the most-efficient animals (4.1\% versus $1.0 \%$ ), with a similar trend observed for Veillonellaceae and Lachnospiraceae, which were increased 3 and 4-fold in the high-efficient group, respectively. To quantitatively and qualitatively measure the ruminal microbial community at lower taxonomic levels (i.e. genus and species level) Carberry et al. (2012) found that inefficient animals had a greater abundance of the genus Prevotella in their rumen community structure. Moreover, the abundance of this genus was highly affected by the nature of the diet offered to cattle, as the presence of Prevotella was almost 4 times greater in the samples from animals consuming a low-forage diet, compared to the ones on a high-forage diet. In contrast, the presence of Fibrobacter succinogenes was 4.5 times greater in the ruminal samples of animals receiving a high-forage diet.

Regarding the overall diversity of rumen microbial populations, although it seems counterintuitive, research has been pointing that ruminal microbiomes with greater richness and diversity are not necessarily associated with improved animal performance $[9,10]$. In fact, a lower richness of microbiome gene content and taxa is strongly correlated with better feed efficiency. The rationalization is that, although the most efficient rumen microbiomes have lower richness and diversity, they produce greater amounts of relevant output metabolites, which are readily available for the animal's energetic needs $[10,11]$. In this context, one important metabolite produced in the rumen, which is normally linked to improved cattle performance is propionate [9]. Thus, efficient microbiomes are in fact less complex, but more specialized in supporting the animal's energy requirements since they produce more relevant compounds, such as propionate.

\section{Conclusion}

As highlighted in this brief review, many differences have been observed in the ruminal microbial population that impact the phenotype relative to animal feed efficiency, as well as the nature of the diet animals are fed. Thanks to new technologies that are now more accessible (especially next-generation DNA sequencing), ruminant nutritionists can have a deeper knowledge of the overall rumen microbiome. Such enhanced knowledge of specific bacterial populations associated with improved animal performance should be coupled with understanding of the biochemical activity of individual microbes of the rumen. Ultimately, these new tools will help nutritionists to feed cattle more efficiently by maximizing the production of important end products such as propionate, which is closely related with feed efficiency. Therefore, next-generation DNA sequencing can guide ruminant nutritionists on selecting in favor of the microorganisms associated with improved animal performance, which in turn can result in greater profitability and sustainability of cattle operations.

\section{References}

1. Brulc JM, Antonopoulos DA, Miller MEB, Wilson MK, Yannarell AC, et al. (2009) Gene-centric metagenomics of the fiber-adherent bovine rumen microbiome reveals forage specific glycoside hydrolases. Proc Natl Acad Sci USA 106: 1948-1953.

2. Russell JB (2002) Rumen microbiology and its role in ruminant nutrition. Ithaca, New York, USA.

3. Hungate RE (1966) The Rumen and its Microbes. New York Academic Press, USA.

4. Bryant M, Robinson IM (1961) Some nutritional requirements of the genus Ruminococcus. Appl Microbiol 9(2): 91-95.

5. McCann JC, Wiley LM, Forbes TD, Rouquette Jr FM, Tedeschi LO (2014) Relationship between the rumen microbiome and residual feed intakeefficiency of Brahman bulls stocked on bermudagrass pastures. PLoS One, 9(3): e91864.

6. Lourenco JM (2017) Utilization of exogenous feed enzymes to enhance creep feeds - in vitro, in vivo, and ruminal microbiome evaluations. $\mathrm{PhD}$ diss. University of Georgia, Athens, Greece.

7. Rosewarne CP, Pope PB, Cheung JL, Morrison M (2014) Analysis of the bovine rumen microbiome reveals a diversity of Sus-like polysaccharide utilization loci from the bacterial phylum Bacteroidetes. J Ind Microbiol Biotechnol 41(3): 601-606.

8. Myer PR, Smith TP, Wells JE, Kuehn LA, Freetly HC (2015) Rumen microbiome from steers differing in feed efficiency. PloS one, 10(6): e0129174.

9. Lourenco JM, DiLorenzo N, Stelzleni AM, Segers JR, Stewart Jr RL (2016) Use of by-product feeds to decrease feed cost while maintaining performance of developing beef bulls. The Professional Animal Scientist 32(3): 287-294.

10. Shabat SKB, Sasson G, Doron-Faigenboim A, Durman T, Yaacoby S, et al. (2016) Specific microbiome-dependent mechanisms underlie the energy harvest efficiency of ruminants. The ISME journal 10(12): 2958.

11. Carberry CA, Kenny DA, Han S, Mc Cabe MS, Waters SM (2012) Effect of phenotypic residual feed intake and dietary forage content on the rumen microbial community of beef cattle. Appl Environ Microbiol 78: 4949-4958. 


\section{Your next submission with Juniper Publishers} will reach you the below assets

- Quality Editorial service

- Swift Peer Review

- Reprints availability

- E-prints Service

- Manuscript Podcast for convenient understanding

- Global attainment for your research

- Manuscript accessibility in different formats ( Pdf, E-pub, Full Text, Audio)

- Unceasing customer service

Track the below URL for one-step submission https://juniperpublishers.com/online-submission.php 